\title{
A New Twist On An Old Tool: Joint Learning With An Innovative Cognitive Writing Tool
}

Jane Mattisson, Kristianstad University, Sweden

Teri Schamp-Bjerede, Blekinge Institute of Technology, Sweden

\begin{abstract}
Our paper introduces a new initiative on a common computer program used as a cognitive tool that facilitates learning with as opposed to learning through technology. The tool, which comprises a modification of a so-called boilerplate, is part of a joint learning system (Kim and Reeves, 2007) in which the tool, learner and activity are equal participators in the learning process. Our focus is on teachers with limited technical expertise. The boilerplate is used as a learning tool specifically aimed at supporting graduate and post-graduate essay writing classes. Teachers have limited time to learn new computer programs and may avoid these because of limited technology skills and inexperience in this area. The cognitive tool assists students with structuring, formatting and grammar. In our presentation, we clarify the special features of the tool, and reflect on the philosophy behind it as well as on its potential for teachers in higher education.
\end{abstract}

Keywords: boilerplate, cognitive learning tool, joint learning system, pedagogy

\section{INTRODUCTION}

riting is an important means of demonstrating one's knowledge in academe. Increasingly, students and teachers are required to present their research findings in English. The strict conventions pertaining to academic English are not always readily identifiable, neither are they easy to master for English-as-aSecond-Language users.

The present article discusses the potential of a so-called boilerplate ${ }^{1}$ as a cognitive learning tool that facilitates learning with as opposed to learning through technology. "Threshold theory" and "troublesome knowledge" constitute our defining concepts. The boilerplate tool is part of a joint learning system (Kim and Reeves, 2007) in which the tool, learner and activity are equal participators in the learning process. Our boilerplate is currently on trial at Kristianstad University in southern Sweden.

In the first half of the article, we consider how the boilerplate enables students to master threshold concepts in academic writing, thereby laying the ground for life-long learning. In the second half, we consider userfriendliness as well as how students and teachers work with computer programs. Selected features of the boilerplate are discussed in relation to their potential both to solve problems for students writing in English and save precious correction time for teachers and tutors. It is our intention in the short term to develop boilerplates for all levels of English studies at Kristianstad University. We also hope in the long term to incorporate the boilerplate in all programs at the University that require students to write in English.

\footnotetext{
${ }^{1}$ This term is used in the publishing industry to denote a template with static elements.
} 


\section{THE BOILERPLATE AS AN ENGLISH-AS-A-SECOND-LANGUAGE LEARNING TOOL}

The boilerplate discussed here was developed specifically for the Level-IV (120 ECTS) English course at Kristianstad University. This course focuses on the teaching and learning of English as a Second Language. As a template containing guidelines, suggestions, and solutions, the boilerplate acts as an aid to students and teachers writing theses as well as papers, reports, and essays in English. Unlike the system already available in Microsoft Word, the boilerplate is tailored to suit specific subjects and types of document. It can be updated on a regular basis, is user-friendly and inexpensive to produce.

Many students are already familiar with the basic functions of the MS Word program, but they do not always choose to take advantage of these when writing. The market is inundated with new devices and synchronous and real-time distance learning, programs for asynchronous and mobile learning, web-based learning, and situated learning, multimodal interaction and augmented devices for learning, as well as content management systems and repositories ( $\mathrm{Li}$, et al, 2008). In contrast, the boilerplate builds on an already existing system with which both teachers and students are already at least partially familiar. It does not entail investment in new equipment and is self-explanatory as users are guided through different options and encouraged to make choices that augment knowledge and enable them to develop their existing knowledge and skills.

As the explanatory diagrams in the second half of the article demonstrate, there are two basic, built-in options in Word when it comes to responding to problems in texts. The first is when a document as opposed to the user, takes advantage of and acts upon options that automatically correct the spelling and grammatical mistakes; this option is known as "hijacking" and is where the program does the work rather than the user. The second option is when Word's built-in spelling and grammar assessor notifies the user. This results in red or green lines appearing beneath the questionable text. Here the user takes advantage of personal knowledge and the special features of the program to correct the mistake; all too often, however, s/he ignores the mistake completely. As neither of these options significantly expands the user's knowledge base, we have implemented a third alternative. This is a modified and expanded version of the first, hijacking option. The database we have created alerts the user to the existence of a problem; s/he receives a message indicating the specific nature of the error, e.g. **There is a problem: grammar** or **There is a problem: spelling** etc. As a consequence, the user is obliged to participate actively in the learning process by utilizing one of the three options.

\section{THRESHOLD CONCEPTS}

Disciplines are characterized by concepts and principles that are subject-specific. These must be mastered as part of the ongoing learning process. Threshold concepts refer to a new way of understanding without which it is difficult to progress within a subject. The thresholds are "conceptual gateways" or "portals" that lead to a particular way of thinking that was previously inaccessible and initially potentially "troublesome" (Meyer \& Land, 2003). As a result, new ways of comprehending, interpreting, or viewing something may emerge that constitute "a transformed internal view of subject matter, subject landscape, or even world view" (Meyer \& Land, 2005, p.379). The boilerplate enables students to acquire threshold concepts related to the discipline itself as well as to the conventions of academic writing in English.

Threshold concepts are "transformative" in that they change the way in which students perceive and practice aspects of their discipline. They are also "irreversible" because once acquired, they are rarely forgotten or "un-learned". Threshold concepts are "integrative" as they allow connections to be made to concepts or knowledge previously unknown or concealed from the student. They are "bounded" in that they help define the boundaries of a subject area. They may also be "troublesome" in that they may involve students in acquiring knowledge that is conceptually difficult or can even be perceived as alien. Following is a brief introduction to how students gain understanding of threshold concepts.

According to Meyer and Land, at the first stage of acquisition students must enter a so-called "luminal space" which can be likened to the experience of adolescents caught between the worlds of the child and adult. If students do not acquire the level of understanding required to "cross a threshold", they will enter a state of "liminality" in which they have only a partial or limited understanding of the concept (Meyer \& Land, 2003). As the 
student acquires a threshold concept, the transformation in understanding may be rapid or occur over a longer period of time. Such a transformation may be exhilarating or disturbing (Palmer, 2001). In crossing a threshold, students experience a shift in feelings, attitudes, emotions, perception, and understanding (Meyer \& Land, 2005; Entwistle, 1981). Language and vocabulary may also be extended as a result of crossing a threshold. There are epistemological and pedagogical differences in acquiring threshold concepts across the disciplines. These relate to the nature of the knowledge existing within a subject and the methods employed to acquire such knowledge.

Threshold concepts may lead to the acquisition of "troublesome knowledge". The latter may consist of tacit knowledge that students find difficult to apply to real-life tasks or for which they have little understanding or evidence (Perkins, 1999). Without acquiring the necessary threshold concepts, however, there is a danger that students will adopt a surface approach to learning in order to complete the educational program (Boustedt, et al., 2007). Threshold concepts not only give students the opportunity to acquire the necessary concepts, they also facilitate understanding of how experts within the field think.

\subsection{Threshold concepts in English as a Second Language}

There are three kinds of threshold concepts in the subject English as a Second Language: methodological, structural and mechanical. In the boilerplate currently being used for the first time on the level-IV course at Kristianstad University, the following methodological threshold concepts relate specifically to the lines along which our specific investigation will be conducted and the criteria for analysis of the selected material. Students are advised in the instructions incorporated in the boilerplate to define theoretical terminology, explain abbreviations, and refer to other research of relevance to the project. They are instructed to explain the method of investigation employed in the study, e.g. interviews, questionnaires, observation etc. The description of the method should be sufficiently detailed that it is possible for others to replicate the study. Students are also instructed to justify their choice of method(s). The instructions in the boilerplate emphasize the importance of referring to secondary sources on research methods in order to validate the student's choice of method.

The structural threshold concepts have been incorporated for introduction, aim, primary/secondary material, method, previous research/theoretical background, analysis and discussion, summary and conclusion, and references and appendices. Each of these is connected to the correct structural font and style that are pre-set for each heading, and explanatory notes are provided, as well as guidelines as to how to express the correct information in a suitably formal style. As the student works on each section, s/he reflects on what is to be included/excluded, the style in which the relevant information is expressed, and the level of detail required for each part. As each section is completed, the student deletes the instructions. Illustrations of how this works in practice are provided below.

The mechanical thresholds incorporated into the boilerplate are extended spelling checkers and punctuation guidelines that indicate incorrect British or American English. These include the use of double or single inverted commas, how to write block quotations, and when and how to use capital letters, colons and semi-colons, italics and bold type. The mechanical threshold concepts include an extended grammar checker that assists students with tense choices, articles, word order, and adjectives and adverbs. Errors of a mechanical kind are marked in the same way as in regular MS Word documents. However, the boilerplate has been extended to include errors not identified by the MS Word grammar checker. These have been selected from previous essays written by students. It is our aim to extend the number of errors accommodated within the boilerplate as our project progresses.

\subsection{Threshold concepts mastered as opposed to learned by rote}

The threshold concepts presented in the boilerplate are pre-requisites for discussing the reference literature that students read in Module A ("The Theory and Practice of English as a Second Language") and Module B ("Testing") of the level-IV "English as a Second Language" course. The instructions in the different sections of the boilerplate guide students in placing the correct information in the right sections. In this way, they gain an overall view of their dissertation as well as develop an understanding of how the different elements relate to one another. Suitable expressions are provided for each section; students must choose the one(s) most suitable for their particular study. In this way, they are encouraged to relate concepts to particular situations and purposes. As our project develops, we shall add concepts and expressions to the boilerplate. Students are aware that they are part of a pilot 
project and have been asked to make note of concepts and functions not currently available in the boilerplate. Suggestions for additions are discussed regularly at seminars where all three supervisors and the students (22) are present. Working with the boilerplate is an ongoing learning process that leads to an increasingly refined product. In this way, we ensure the best possible level of user-friendliness. Our boilerplate will undergo thorough usability testing, a feature which is all too often forgotten when developing a new or - as in our case - modifying an existing technological product. ${ }^{2}$

\section{USER-FRIENDLINESS IN HUMAN-COMPUTER INTERACTION}

Using text editors is part of the standard student and teacher knowledge base, yet higher-level functions such as those the boilerplate utilizes can be perplexing if one does not consider the knowledge level of the user. The boilerplate project takes into consideration pedagogical issues that both teachers and students face when they are required to use computer programs in their courses. When using any technological program, it is important to reflect upon Human-Computer-Interaction (HCI) issues such as usability and user-friendliness. One area that Usability Studies investigates is how easy a particular program is to use. Many users will never read an instruction manual; they utilize previous experience in order to help them comprehend and use the program. The clarity of the program's design is particularly important with regard to the ease with which the user is able to interact with/use the program. As discussed above, both students and teachers use technology for different purposes and users are guided by previous experience. Both teachers and students have expectations of not only which desktop icons should be visibly accessible but also where they should be located. A case in point is the operating systems MS XP and MS Vista. Both of these have the traditional Start icon in the lower left hand corner, and the clock in the lower right hand corner.

All computer users have experience as their main guide. Programmers and technology majors with a broader level of knowledge and experience will readily identify familiar icons in unfamiliar places. Their level of experience will also enable them to recognize unfamiliar icons where familiar ones are expected (Nielsen, 2009). As a result, when students and teachers use a program, they will anticipate these icons based on previous experience. By utilizing a user-friendly program that is familiar to both teachers and students there is more focus on the task, as there is no need to learn a new and unfamiliar program.

\subsection{Methodological Elements}

As seen in Images 1, 2 and 3, the addition of instructions within the boilerplate creates a direct connection to the content required for each section. Black is reserved for actual essay content while blue denotes boilerplate instructions; in this way, students are able to differentiate between texts that are to be kept and texts that are directly related to the boilerplate instructions. Image 1 presents some of the basic boilerplate instructions to be removed before final submission.

\footnotetext{
${ }^{2}$ This point is made by among others Jay Buzhardt and Linda Heitzman-Powell (2005) in "Stop blaming the teachers: the role of usability in bridging the gaps between educators and technology". Buzhardt and Heitzman-Powell argue that "an infinite amount of training and improvement to schools' ability to implement change will do little to overcome the poor usability of a software application or website. Although technology developers are responsible for optimizing the usability of their products, the education community (teachers, administrators, [...], legislators etc.) must be responsible purchasers and consumers of educational technology. This community has a right to expect and demand technology that has undergone rigorous usability testing and has data to support its use in educational settings", Electronic Journal for the Integration of Technology in Education 4, 13- 29 (pp. 14-15). As part of the continuation of our boilerplate project, we intend to focus on usability: how do we enable teachers and students to implement the boilerplate in their studies and articles? How can the boilerplate be made more userfriendly? To answer these questions, we intend to conduct a separate study on how students and teachers learn to use the boilerplate.
} 
Image 1. Boilerplate Instructions

Boilerplate Instructions: English Level-IV students
1) All formatting has been pre-styled to be used specifically with WORD 2003:
please do not change it!
2) Open the FORMAT Tab in WORD 2003, and choose "Styles and Formatting". The
options will appear on the right hand side of the Word document.
A) How to change and apply the formatting possibilities:
$\quad$ Highlight the text and click the appropriate option:
$\quad$ Heading, Body Text, Long Quotations, References, etc...
How to:
Replace the sample information with your own. Start by filling in the title page and
your essay information (see Image 4).

Incorporating specific methodological instructions in the boilerplate itself means that they are always on hand. Only when the tasks have been completed are they deleted (Images $2 \& 3$ ).

\section{Image 2. Student Guidelines}

1. Intro duction
State your area of study, simply and clearly. Give a brief introduction to the background of the
problem investigated so that the reader understands the topie of the essay and why this is relevant.
Length: maximum 1 page.
1.1.Aim
Write a detailed description of what you intend to do in your essay, including what aspects you
are going to investigate. The aim is the foundation of your essay, All material you collect, the
methods you use, and the aspects you analyze must be in line with your aim. Make your aim
specific and limited. Length: maximum a few lines.
1.2 Material
A presentation of the primary materials used in your study, t.e. materials from your field studies,
or the teachingtesting materials you are going to analyze. Describe the materials you are using in
detail, how you collected them, and what motivated your choice of materials data. Remember, for
ethical reasons all parties are to remain anorymous.
Length: this will be as short or as long as your material requires.


Image 3. Information regarding sections and sub-sections

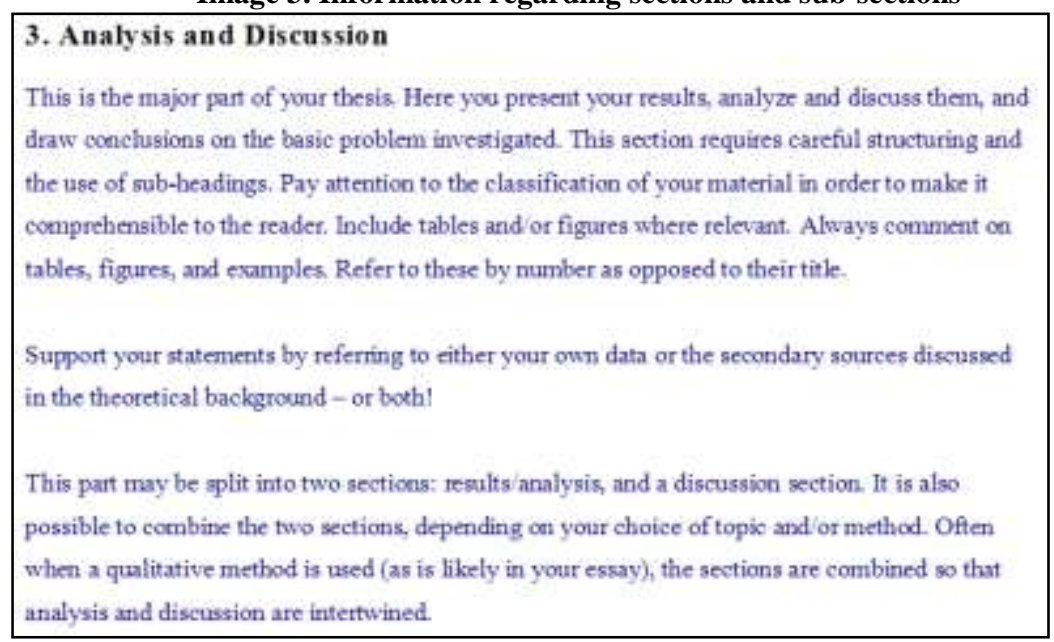

\subsection{Structural elements}

An essay's title page needs to contain visual connections to a professional document (Image 4). As seen here, the layout is set so that there is a styled space between title and author information. Differences as to manual line breaks and paragraph breaks are also visible. By using a dynamic table of contents (Image 5), the user has the benefit of the program to aid and keep track of all changes regarding page numbers and chapters, multiple subchapters, references, and appendices. Most essays require listing and keeping track of different data. The most widely accepted methods are bulleted lists and numbered/alphabetical lists, as seen in Image 6. The bulleted list is used for generic points, while numbered/alphabetical lists are used where sub-sections need to be included.

Image 4. Title Page - Formatting View

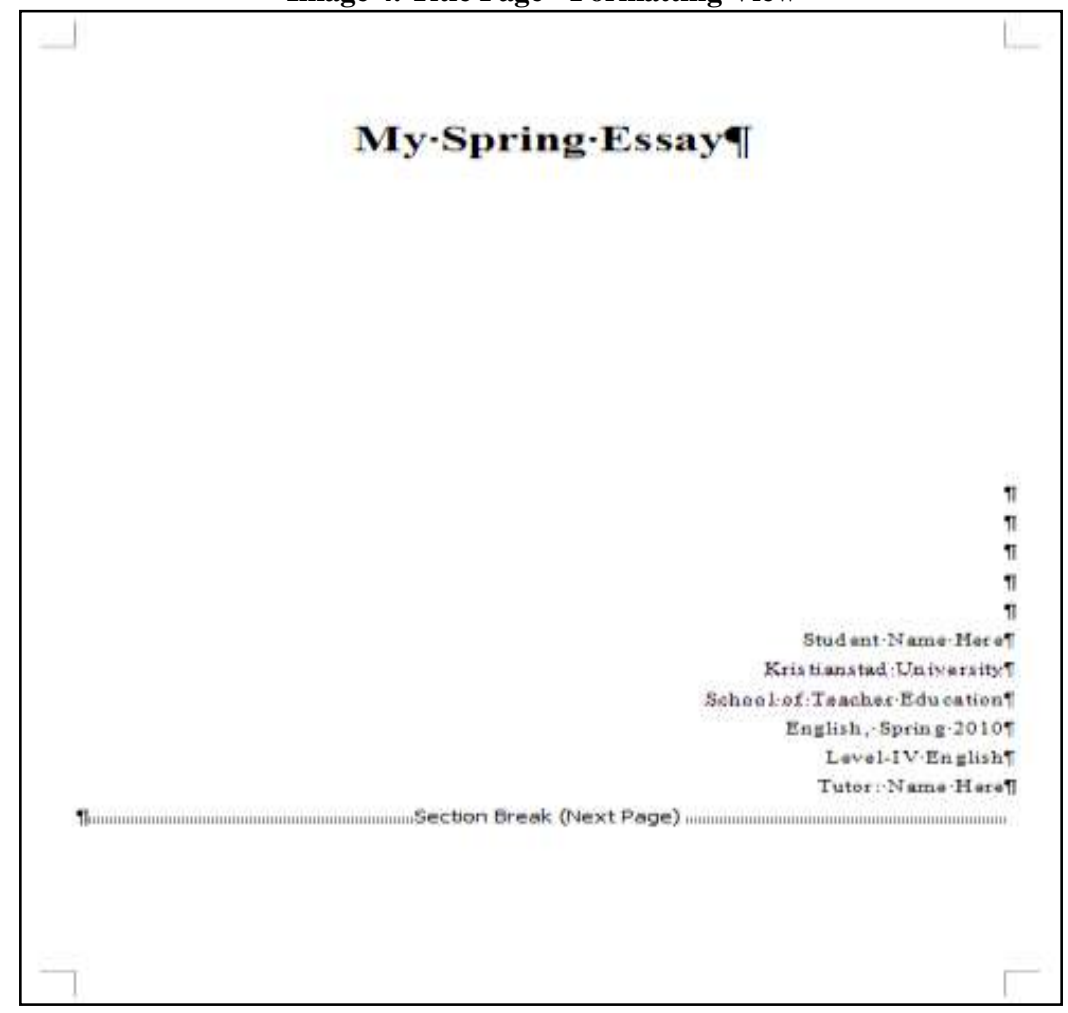


Image 5. Structural Formatting for Dynamic Table of Contents

\begin{tabular}{|c|}
\hline $\begin{array}{l}\text { Table of Contents } \\
\text { Acknowledgemens }\end{array}$ \\
\hline 1. Innoduction \\
\hline $1.1 \mathrm{Aim}$ \\
\hline 12 Mtaterial \\
\hline 13 Method_ \\
\hline 2. Previous Research / Theorvetical Background \\
\hline $\begin{array}{l}\text { 2.1 Previous Research } \\
21,1 \text { Badkgound Statistici }\end{array}$ \\
\hline 2.2 Theoretical Background \\
\hline 3. Anabysis and Discussion. \\
\hline 4. Summary and Conclusion \\
\hline Refervences \\
\hline Appendices \\
\hline $\begin{array}{l}\text { Appendia } 1 \\
\text { Appendix } 2-\end{array}$ \\
\hline
\end{tabular}

Image 6. Structural formatting: bulleted and numbered lists

- Example-Bulleted Lists

- Corpus Information

- Thesaurus

- Encoding

1. Example - Numbered Lists

2. Vocabulary Assessment

2.1. Age

2.2. Gender

3. Class Setting

3.1. Outdoors

3.1.1. Group

3.1.1.1. Gender

3.1.1.1.1. Age

4. Image Word Formation

\subsection{Mechanical elements}

These elements are noted and added to the boilerplate's database of problems and solutions. As seen in Tables A and B below, " 1 " is the questionable element, " 2 " is an automatic "high-jacked" correction, and " 3 " is a learning option we have implemented where the student is informed of the error and must correct the problem him/herself. 
Table A: Correcting Grammar

\begin{tabular}{|l|l|}
\hline 1. & Last year we go on vacation. \\
\hline 2. & Last year we went on vacation. \\
\hline 3. & Last year we **GRAMMAR CHECK - Verb Tense** on vacation. \\
\hline
\end{tabular}

Table B: Correcting Spelling

\begin{tabular}{|l|l|}
\hline 1. & When learning new teknologies, it is best to be careful. \\
\hline 2. & When learning new technologies, it is best to be careful. \\
\hline 3. & When learning new $* *$ SPELLING CHECK - Word $* *$ it is best to be careful. \\
\hline
\end{tabular}

Image 7 is a screenshot from MS Word showing a recurring error made by many students, i.e. the use of and as the first word in a new sentence. By adding specific components of commonplace errors and subsequent notices for the students, it is hoped that the standard of the language used in essays will improve. Image 8 is a screenshot from MS Word that shows how we have modified the "hijacking option" and replaced it with our own informative notice that requires an active response from the student to correct the mistake.

Image 7 Grammatical Error - "And"

In this process, the relevant points and links become more overt, and the irrevelant ones are supressed, until finally one word wins out over the numerous others that are activated. And this is also true for word recognition.

Image 8 Database Correction Notice

In this process, the relevant points and links become more overt, and the irrevelant ones are supressed, until finally one word wins out over the numerous others that are activated **GRAMMAR CHECK: Use of "AND"** this is also true for word recognition.

\section{CONCLUSION}

The boilerplate discussed above has enormous potential for a wide variety of disciplines due to its flexibility, user-friendliness, and low cost. It is also highly democratic as it enables the tool, learner, and activity to participate in the learning process on equal terms. The current pilot study of the boilerplate at Kristianstad University has yielded many positive results, as demonstrated in the regular seminars with the students, questionnaires distributed throughout the course and in feedback from individual students via e-mail. Among comments received is that students have appreciated the comprehensive nature and clarity of the instructions in the boilerplate, the pre-formatting of margins, headings, etc. and assistance with remedying errors such as grammar, spelling, punctuation and style. Students' written English has improved in terms of both accuracy and style. The authors warmly welcome questions and comments by e-mail: jane.mattisson@hkr.se and teri.schampbjerede@bth.se.

\section{REFERENCES}

1. Boustedt, J., Eckerdal, A, McCartney, R., Moström, J.E., Ratcliffe, M., Sanders, K. \& Zander, C. (2007) 'Threshold concepts in computer science: do they exist and are they Useful?' Proceedings of the Special Interest Group on Computer Science Education (SIGCSE), 7-10 March, Covington, Kentucky. New York, NY: ACM, pp.504-508.

2. Buzhardt, J. \& Heitzman-Powell, L. (2005) 'Stop blaming the teachers: the role of usability in bridging the gaps between educators and technology', Electronic Journal for the Integration of Technology in Education, 4, pp.13-29, CiteSeerX [Online]. Available from: http://ejite.isu.edu/ (Accessed: 30 April 2009). 
3. Entwistle, N. (1981) 'Styles of Learning and Teaching: An Integrated Outline of Educational Psychology for Students', Teachers and Lecturers. Great Britain: David Fulton Publishers.

4. Kim, B. \& Reeves, T. (2007) 'Reframing research on learning with technology: in search of the meaning of cognitive tools', Instructional Science, 35(3), pp. 207-256, IngentaConnect [Online]. DOI: 10.1007/s11251-006-9005-2 (Accessed: 3 May 2010).

5. Li, Q., Lau, R. W., Shih, T. K., and Li, F. W. (2008) 'Technology supports for distributed and collaborative learning over the internet'. ACM Transactions on Internet Technology (TOIT), 8 (2), pp.1-24, ACM [Online]. Available from: http://doi.acm.org/10.1145/1323651.1323656 (Accessed: 30 April 2010).

6. Meyer, J. \& Land, R. (2005) 'Threshold concepts and troublesome knowledge (2): Epistemological considerations and a conceptual framework for teaching and Learning ', Higher Education, 49 (3) pp.373388, IngentaConnect [Online]. Available from: http://www.ingentaconnect.com/ (Accessed: 30 April 2010).

7. Meyer, J. \& Land R. (2003) 'Threshold concepts and troublesome knowledge: Linkages to ways of thinking and practicing within the disciplines', in Improving Student Learning: Improving Student Learning Theory and Practice - Ten Years On, ed. Rust, C., Oxford Centre for Staff and Learning Development (OCSLD), Oxford, pp.412-424.

8. Nielsen, J. (1994) 'Enhancing the explanatory power of usability heuristics', Proceedings of the SIGCHI Conference on Human Factors in Computing Systems: Celebrating interdependence (CHI '94), 24-28 April, Boston, Massachusetts. Adelson, B., Dumais, S. \& Olson, J., eds. New York, NY: ACM, pp.152-158.

9. Palmer, R. (2001) 'The Liminality of Hermes and the Meaning of Hermeneutics', Richard E. Palmer Personal Website [Online]. Available from: http://www.mac.edu/faculty/richardpalmer/liminality.html (Accessed: 30 April 2010).

10. Perkins, D. (1999) 'The many faces of constructivism ', Educational Leadership 57 (3), pp.115-133, [Online]. Available from: http://web.ebscohost.com (Accessed: 30 April 2010). 
NOTES 